
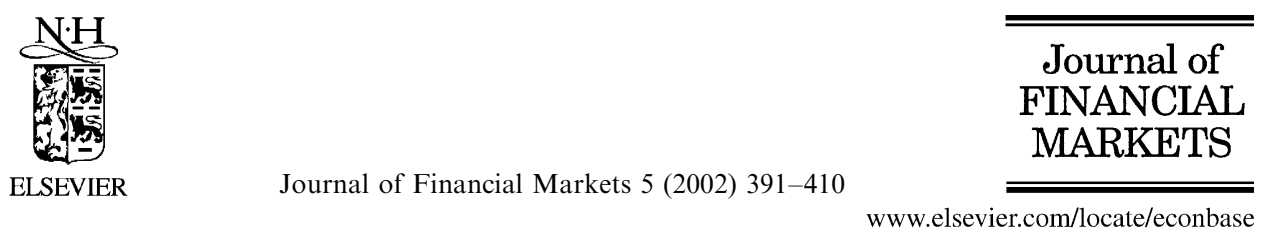

\title{
Tick size and quote revisions on the NYSE ${ }^{\text {th }}$
}

\author{
Kee H. Chung ${ }^{\mathrm{a}, *}$, Chairat Chuwonganant ${ }^{\mathrm{b}}$ \\ ${ }^{a}$ The M\&T Chair in Banking and Finance, Department of Finance and Managerial Economics, \\ School of Management, The State University of New York (SUNY) at Buffalo, Buffalo, \\ NY 14260, USA \\ ${ }^{\mathrm{b}}$ Indiana University-Purdue University at Fort Wayne, Fort Wayne, IN 46805, USA
}

\begin{abstract}
In this paper, we analyze how tick size affects quote revisions on the NYSE and whether pre-decimalization tick sizes $(\$ 1 / 8$ and $\$ 1 / 16)$ were binding constraints on specialists' spreadand price-quote decisions. We find that the number of quote revisions that involve changes in the spread increased dramatically after the tick-size reduction in 1997. The number of spreadquote revisions is smaller for stocks with lower prices and larger volumes during both the pre and post tick-size change periods. We interpret this result as evidence that the minimum price variation is a binding constraint on absolute spreads even after the tick-size reduction, especially for low-price and/or large-volume stocks. The number of quote revisions that involve changes in the spread (depth) is largest (smallest) during the early hour of trading, suggesting that the minimum price variation is least likely to be the binding constraint on spreads during the early hour of trading. These results suggest that decimalization is likely to further reduce price rigidity and increase price competition. Consistent with this expectation, we find a significant increase in the frequency of spread- and price-quote revisions after decimalization. (C) 2002 Elsevier Science B.V. All rights reserved.
\end{abstract}

JEL classification: $\mathrm{G} 14$

Keywords: Specialists; Spreads; Depths; Liquidity providers; Minimum price variation

\footnotetext{
${ }^{\text {is }}$ The authors thank an anonymous referee, Avanidhar Subrahmanyam (the editor), Pinaki Bose, Thomas McInish, William Smith, and Robert Wood for valuable comments and helpful discussions. The authors are solely responsible for the contents of the paper.

*Corresponding author. Tel.: + 1-716-645-3262; fax: + 1-716-645-2131.

E-mail address: keechung@acsu.buffalo.edu (K.H. Chung).
} 


\section{Introduction}

The minimum price variation (tick size) on the New York Stock Exchange (NYSE) was reduced from $\$ 1 / 8$ to $\$ 1 / 16$ on June 24,1997 . In this study, we examine how the minimum price variation affects quote revisions on the NYSE. Specifically, we analyze how specialists and other liquidity suppliers use the spread and depth (i.e., size) in quote revisions, and whether the tick-size change has altered their quote revision behavior.

Although previous studies examine the impact of the tick-size change on dealer/ specialist quotes, the primary focus of these studies has been how the tick-size reduction affected quoted spreads and depths. For example, Harris (1994) predicts that a reduction in the minimum price variation will cause spreads to narrow and depths to decline. Ahn et al. (1996) examine the change in liquidity when the Amex reduced tick size and find that both the spread and depth declined with the smaller tick size. Bacidore (1997) and Porter and Weaver (1997) examine the impact of the tick-size change on liquidity for stocks listed on the Toronto Stock Exchange (TSE). Bacidore shows that the liquidity change for TSE-listed stocks resulting from the tick-size reduction is similar to that reported in Ahn et al. (1996) for Amex-listed stocks. Similarly, Porter and Weaver show that the smaller tick size results in smaller depths and lower execution costs for low-price and large-volume stocks.

Simaan et al. (1998) study the quotation behavior of Nasdaq dealers following the tick-size change. The authors find that Nasdaq dealers continue to avoid odd ticks, but traders entering orders on Electronic Communication Networks (ECNs) do not exhibit such behavior. Their findings indicate that ECNs frequently establish the inside market quote and reduce trading costs for the public. Bollen and Whaley (1998), Goldstein and Kavajecz (2000) and Van Ness et al. (2000) examine the impact of the tick-size change on the spread and depth of NYSE stocks. These studies find that both quoted spreads and depths declined after the tick size changed from eighths to sixteenths. Goldstein and Kavajecz suggest that the combined effect of smaller spreads and reduced cumulative depths in the book has helped liquidity demanders trading small orders while hurting those trading large orders. Griffiths et al. (1998) examine the impact of the tick-size change on the market-making behavior on the TSE and conclude that the tick-size reduction benefits the public.

Although previous studies show that the tick-size change has significant effects on the spread and depth of NYSE-listed stocks, there are still many unanswered questions on how the tick-size change has affected the quote-setting process. How often do specialists and other liquidity providers make quote revisions involving a change only in the spread or depth? ${ }^{1}$ How has quote revision behavior been affected by the change in tick size? Is the reduced tick size (i.e., \$1/16) small enough to give liquidity providers sufficient freedom in their quote decisions? Or does the tick size still pose a restriction on quote decisions for certain stocks? We believe empirical

\footnotetext{
${ }^{1}$ Several studies suggest that market makers use both the spread and depth as strategic choice variables for liquidity management (see, e.g., Ye, 1995; Kavajecz, 1996, 1999; Kavajecz and Odders-White, 1999).
} 
evidence regarding these issues is useful for assessing the possible impact of decimal pricing on market quality.

In this study, we perform a before-and-after analysis of quote revision behavior surrounding the tick-size change using a sample of 2,223 NYSE-listed stocks. Specifically, we examine how price discreteness affects quote revisions on the NYSE and whether pre-decimalization tick sizes (i.e., $\$ 1 / 8$ and $\$ 1 / 16$ ) were binding constraints on absolute spreads. Although discreteness is likely to affect both the spread and depth revisions, we conjecture that price discreteness might have a greater effect than size discreteness to the extent that minimum price variation is more frequently the binding constraint than minimum quantity variation. We provide empirical evidence regarding this issue using data during both the pre and post tick-size change periods. In addition, we analyze intraday variation in quote revisions and thereby shed further light on whether the minimum price variation affects the relative use of spreads and depths over different times of the day.

Our results show that the frequency of quote revisions that involve changes in the spread is smaller than the frequency of quote revisions that involve changes in the depth, despite the fact that the former increased dramatically after the tick-size reduction. Our cross-sectional regression analyses show that the proportion of quote revisions that involve changes in the spread is smaller for stocks with lower prices and larger volumes during both the pre and post tick-size change periods. We also find that the minimum tick size affects intraday variation in the frequency of spread revisions during both periods. We interpret these results as evidence that the minimum price variation is the binding constraint on absolute spreads even after the tick-size reduction. Based on these findings, we predict that decimal pricing will further reduce price rigidity and increase price competition. We find evidence in support of this prediction.

The paper is organized as follows. Section 2 discusses our data and methodology. Section 3 examines how quote revision behavior is related to the attributes of stocks and tests whether the minimum price variation poses a restriction on quote revision. Section 4 analyzes the intraday pattern of quote revisions. Section 5 examines the effect of decimal pricing on the relative frequency of spread- and depth-quote revisions. Section 6 provides a brief summary and concluding remarks.

\section{Data and methodology}

\subsection{Data source and sample selection}

We obtain data for this study from the NYSE's TAQ database. We use the trade and quote data during 30 trading days immediately before and after the date (June $24,1997)$ on which the minimum price variation changed from $\$ 1 / 8$ to $\$ 1 / 16$. To secure a study sample of reasonably active stocks, we omit stocks with less than 10 transactions per day (i.e., 600 transactions during the entire study period) from the initial sample of 2,725 NYSE stocks. This leaves us with a sample of 2,261 stocks. We find that 36 of these remaining stocks had a bid price of less than $\$ 1$ at least once 
during the study period. We omit these stocks from our study sample because the minimum price variation becomes $\$ 1 / 32$ when the bid price drops below $\$ 1$. We also delete two additional stocks from the study sample: one with a spread of greater than $\$ 5$ during the entire study period and the other with a price in excess of $\$ 1,000$. This leaves us with a total of 2,223 stocks as our final study sample.

In addition, we omit the following trades and quotes to minimize data errors: ${ }^{2}$

1. quotes if either the ask price (size) or the bid price (size) is less than or equal to zero;

2. quotes if the bid-ask spread is greater than $\$ 5$ or less than zero;

3. trades and quotes if they are out of time sequence, involve an error, or involve a correction;

4. quotes associated with trading halts or designated order imbalance;

5. before-the-open and after-the-close trades and quotes;

6. trades if the price or volume is less than or equal to zero;

7. trade price, $p_{t}$, if $\left|\left(p_{t}-p_{t-1}\right) / p_{t-1}\right|>0.10$;

8. ask quote, $a_{t}$ if $\left|\left(a_{t}-a_{t-1}\right) / a_{t-1}\right|>0.10$; and

9. bid quote, $b_{t}$, if $\left|\left(b_{t}-b_{t-1}\right) / b_{t-1}\right|>0.10$.

We report several attributes of our study sample of 2,223 stocks in Table 1 . The average share price before the tick-size change is $\$ 31.19$ and the average price after the change is $\$ 32.71$. (Share price is measured by the mean daily closing price during the study period.) The average trade size (in number of shares) and the average daily number of transactions are 2,076 and 81.23, respectively, before the tick-size change and 1,934 and 97.09 after the tick-size change. The average standard deviation of daily returns is 0.0185 before the tick-size change and 0.0201 after the change. The results of paired comparison $t$-tests show that the number of trades has gone up and the average trade size has declined significantly since the tick-size change. Consistent with the finding of Goldstein and Kavajecz (2000) and Van Ness et al. (2000), we find a significant decrease in both the spread and depth (the ask size plus the bid size) after the tick-size change.

\subsection{Quote revision classification}

To examine how NYSE specialists jointly utilize spreads and depths as means of liquidity management, we analyze how they change their spreads and depths between two consecutive quotes. While we label quotes from the TAQ database as specialist quotes for expositional convenience, we note that these quotes reflect not only the trading interests of specialists but also limit orders placed by outsiders. ${ }^{3}$ Oftentimes,

\footnotetext{
${ }^{2}$ See Huang and Stoll (1996) for a similar method. Our filter 7 eliminates 23 trades from the pre tick-size change data and 100 trades from the post tick-size change data and filters 8 and 9 eliminate 126 quotes from the pre tick-size change data and 344 quotes from the post tick-size change data.

${ }^{3}$ Harris and Hasbrouck (1996) show that limit orders represent about $54 \%$ of all orders submitted through SuperDOT. Similarly, Chung et al. (1999) find that about $20 \%$ of quotes in the NYSE's TORQ (Trades, Orders, Reports, and Quotes) database originate from traders on both the bid and ask sides without any direct participation of specialists. Chung et al. also find that in more than two-thirds of TORQ quotes, at least one side of the quote originates exclusively from limit order traders.
} 
Descriptive statistics

This table shows the attributes of our study sample of 2,223 stocks before and after the tick-size change. Share price is measured by the mean daily closing price. Number of trades is the average daily number of transactions. Trade size is the average transaction size in number of shares. Return volatility is measured by the standard deviation of daily returns. Spread is the average quoted dollar spread (the ask price - the bid price). Depth is the average quoted depth (the bid size + the ask size). We also show the results of paired comparison $t$-tests on the equality of the mean between the two periods.

\begin{tabular}{|c|c|c|c|c|c|c|c|c|c|}
\hline & \multicolumn{3}{|c|}{ Before the tick-size change } & \multicolumn{3}{|c|}{ After the tick-size change } & \multicolumn{3}{|c|}{$\begin{array}{l}\text { Testing the difference in the mean } \\
\text { between the two periods }\end{array}$} \\
\hline & Mean & Median & $\begin{array}{l}\text { Standard } \\
\text { deviation }\end{array}$ & Mean & Median & $\begin{array}{l}\text { Standard } \\
\text { deviation }\end{array}$ & $\begin{array}{l}\text { Difference } \\
\text { (after - before) }\end{array}$ & $t$-value & $p$-value \\
\hline Share price $(\$)$ & 31.19 & 25.68 & 23.85 & 32.71 & 27.32 & 24.46 & 1.52 & 12.17 & 0.0001 \\
\hline Number of trades & 81.23 & 37.07 & 133.53 & 97.09 & 44.93 & 152.21 & 15.86 & 19.61 & 0.0001 \\
\hline Trade size ${ }^{a}$ & 2,076 & 1,797 & 1,281 & 1,934 & 1,669 & 1,241 & -142 & -7.39 & 0.0001 \\
\hline Spread (\$) & 0.1852 & 0.1791 & 0.0424 & 0.1557 & 0.1484 & 0.0601 & -0.0295 & -51.34 & 0.0001 \\
\hline Depth $^{\mathrm{b}}$ & 230.5 & 94.36 & 367.7 & 138.9 & 67.72 & 229.0 & -91.6 & -22.83 & 0.0001 \\
\hline
\end{tabular}

${ }^{\mathrm{a}}$ In number of shares.

${ }^{\mathrm{b}}$ In round lots. 
specialist quotes include the trading interests of floor brokers as well. Hence, our use of the term 'specialists' should be interpreted broadly to include all other suppliers of liquidity.

We classify each pair of consecutive spread quotes into one of three quote revision groups: decrease in spread (-), no change in spread $(0)$, and increase in spread $(+)$. Similarly, we classify every pair of consecutive depth quotes into one of three quote revision groups: decrease in depth $(-)$, no change in depth (0), and increase in depth $(+)$. By following this procedure, we classify each quote change into a quote revision class (QRC hereafter) (S,D), where $\mathrm{S}(\mathrm{S}=-, 0,+)$ represents the quote revision group for the spread and $\mathrm{D}(\mathrm{D}=-, 0,+)$ represents the quote revision group for the depth. ${ }^{4}$ For example, if a quote change involves an increase in the spread and a decrease in the depth, we classify the quote change into QRC $(+,-)$. Similarly, if a quote change involves only a decrease in the spread (and no change in the depth), we classify the quote change into QRC $(-, 0)$.

\subsection{Impact of the tick-size change on quote revision behavior}

To examine how the tick-size change has affected the relative frequency of quote revisions involving changes in the spread (depth), we compare the proportion of quote revisions that involve changes in the spread (depth) during the pre tick-size change period with the proportion during the post tick-size change period. Table 2 shows that before the tick-size change, only $28.21 \%$ of quote revisions involve changes in the spread $(\mathrm{SPCH})$ [i.e., QRCs $(-,-),(-, 0),(-,+),(+,-),(+, 0)$, and $(+,+)$ ], while $75.38 \%$ of quote revisions involve changes in the depth (DPCH) [i.e., QRCs $(-,-),(-,+),(0,-),(0,+),(+,-)$, and $(+,+)]$. After the tick-size reduction, however, the proportion of quote revisions that involve changes in the spread went up by $12.54 \%$ to $40.75 \%$, while the proportion of quote revisions involving changes in the depth went up only slightly to $76.51 \%$.

Similarly we find that, before the tick-size reduction, $5.32 \%$ of quote revisions involve changes only in the spread (SPONLY) [i.e., QRCs $(-, 0)$ and $(+, 0)$ ] and $52.48 \%$ of quote revisions involve changes only in the depth (DPONLY) [i.e., QRCs $(0,-)$ and $(0,+)]$. After the tick-size reduction, however, SPONLY increased to $8.81 \%$, while DPONLY dropped by $7.91 \%$ to $44.57 \%$. The results of paired comparison $t$-tests indicate that these differences are statistically significant.

Although our results show that liquidity providers revise their depth quotes more frequently than spread quotes during both the pre and post tick-size change periods, we note that they can revise their price quotes without changing spread quotes through parallel shifts in the bid and ask prices. In this case, the relative frequency of spread-quote revisions can be an inaccurate measure of price rigidity. To examine

\footnotetext{
${ }^{4}$ Although our quote classification utilizes only the direction of a given quote change (instead of the actual size of the change), our method does not lose much information associated with spread-quote revisions since most spread-quote revisions involve changes of one tick in the bid and/or ask prices. We note that our method utilizes only a part of information contained in depth-quote revisions because most depth-quote revisions are greater than one round lot.
} 
this possibility, we analyze how liquidity providers revise their bid and ask prices relative to the bid and ask depths. We show the results in panels $\mathrm{B}$ and $\mathrm{C}$ of Table 2 . The results show that the frequency of quote revisions involving changes in the bid (ask) price increased after the tick-size reduction. Before the tick-size change, $18.32 \%$ of quote revisions involve changes in the bid price $(\mathrm{BPCH})$, while the corresponding figure is $24.21 \%$ after the tick-size change. We find, however, that depth-quote revisions remain more frequent than price-quote revisions after the ticksize reduction.

Our empirical results indicate that although specialists and other liquidity providers revise the depth more frequently than the spread during both the pre and post tick-size change periods, their use of the spread has increased dramatically since the tick-size change. One possible explanation for this result is price discreteness. Before the tick-size change, the minimum price variation is $\$ 1 / 8$ for stocks with a bid price of $\$ 1$ or higher, while depths are quoted in multiples of 100 shares. Although discreteness is likely to affect both the spread and depth, price discreteness might have a greater effect than depth discreteness to the extent that minimum price variation is more frequently the binding constraint than minimum quantity variation. As a result, liquidity providers may end up using the depth more frequently than the spread for their liquidity management. After the tick size is reduced to $\$ 1 / 16$, however, the extent to which minimum price variation is the binding constraint on spreads becomes smaller, and thus specialists and other liquidity suppliers rely more on the spread than they did before the tick-size reduction.

To confirm whether the increased use of the spread after the tick-size reduction is indeed due to the change in the binding constraint, we calculate the proportion (PMIN) of spread quotes that are equal to the tick size (see Table 2). Note that the proportion of spread quotes that are equal to $\$ 1 / 8$ before the tick-size change is $60.32 \%$, whereas the proportion of spread quotes that are equal to $\$ 1 / 16$ after the change is only $32.96 \%$. We find similar results from the percentage of time (PTIMEMIN) that the quoted spread is equal to the minimum price variation. These results suggest that the probability that the minimum price variation is the binding constraint on absolute spreads declined significantly after the tick-size reduction. Hence, the increased use of the spread after the tick-size reduction appears to be driven in part by the fact that the smaller tick size poses less restriction on specialists' spread-quote decisions. $^{5}$

\footnotetext{
${ }^{5}$ There are several other possible explanations for the result. One possible explanation is the limit order display rule, which requires market makers to update depth quotes whenever they receive customer limit orders that add size to their quotes at the inside price. Note also that a trade can consume a portion of the quoted depth without changing the posted price. Although NYSE specialists have little discretion over spread quotes because of competitive pressures from other liquidity suppliers (e.g., floor traders and regional exchanges), they have much more control over their depth quotes. Hence, the greater frequency of depth revisions relative to spread revisions documented in the present study may also be attributed at least in part to specialists' greater control over their depth quotes.
} 
Table 2

Proportion of quote changes involving changes in the spread, depth, bid price, bid size, ask price, and ask size

This table shows the mean (median) values of SPCH, SPONLY, DPCH, DPONLY, BPCH, BPONLY, BSCH, BSONLY, APCH, APONLY, ASCH, ASONLY, PMIN, and PTIMEMIN before and after the tick-size change and the results of paired comparison $t$-tests on the equality of the mean between the two periods. SPCH is the proportion of quote revisions involving changes in the spread, DPCH is the proportion of quote revisions involving changes in the depth, SPONLY is the proportion of quote revisions involving changes only in the spread, and DPONLY is the proportion of quote revisions involving changes only in the depth. Similarly, BPCH $(\mathrm{APCH})$ is the proportion of quote revisions involving changes in the bid (ask) price, $\mathrm{BSCH}(\mathrm{ASCH})$ is the proportion of quote revisions involving changes in the bid (ask) size, BPONLY (APONLY) is the proportion of quote revisions involving changes only in the bid (ask) price, and BSONLY (ASONLY) is the proportion of quote revisions involving changes only in the bid (ask) size. PMIN is the proportion of spread quotes that are equal to the tick size. PTIMEMIN is the percentage of time that spread quotes are equal to the tick size.

\begin{tabular}{lllll}
\hline $\begin{array}{l}\text { Before the tick-size } \\
\text { change }\end{array}$ & $\begin{array}{l}\text { After the tick-size } \\
\text { change }\end{array}$ & $\begin{array}{l}\text { Difference } \\
\text { (after - before) }\end{array}$ & $t$-value & $p$-value \\
\hline
\end{tabular}

A. Spread-depth revisions

$\begin{array}{lcccrr}\text { SPCH } & 28.21 \%(27.60 \%) & 40.75 \%(40.85 \%) & 12.54 \%(13.25 \%) & 31.23 & 0.0001 \\ \text { SPONLY } & 5.32 \%(3.35 \%) & 8.81 \%(7.31 \%) & 3.49 \%(3.96 \%) & 17.99 & 0.0001 \\ \text { DPCH } & 75.38 \%(77.61 \%) & 76.51 \%(77.84 \%) & 1.13 \%(0.23 \%) & 3.48 & 0.0005 \\ \text { DPONLY } & 52.48 \%(52.81 \%) & 44.57 \%(44.18 \%) & -7.91 \%(-8.63 \%) & -20.94 & 0.0001 \\ \text { PMIN } & 60.32 \%(59.67 \%) & 32.96 \%(28.68 \%) & -27.36 \%(-30.99 \%) & -45.63 & 0.0001 \\ \text { PTIMEMIN } & 61.73 \%(61.99 \%) & 33.39 \%(27.97 \%) & -28.34 \%(-34.02 \%) & -42.91 & 0.0001\end{array}$

B. Bid price-bid depth revisions

\begin{tabular}{lccrrr} 
BPCH & $18.32 \%(17.35 \%)$ & $24.21 \%(23.82 \%)$ & $5.89 \%(6.47 \%)$ & 21.78 & 0.0001 \\
BPONLY & $4.35 \%(2.72 \%)$ & $6.27 \%(5.09 \%)$ & $1.92 \%(2.37 \%)$ & 12.90 & 0.0001 \\
BSCH & $41.06 \%(41.94 \%)$ & $40.46 \%(40.76 \%)$ & $-0.60 \%(-1.18 \%)$ & -2.76 & 0.0059 \\
BSONLY & $27.09 \%(26.92 \%)$ & $22.52 \%(22.11 \%)$ & $-4.57 \%(-4.81 \%)$ & -20.28 & 0.0001 \\
C. Ask price-ask depth revisions & & & & \\
APCH & $18.13 \%(16.91 \%)$ & $24.62 \%(24.01 \%)$ & $6.49 \%(7.1 \%)$ & 23.42 & 0.0001 \\
APONLY & $4.19 \%(2.40 \%)$ & $6.01 \%(4.67 \%)$ & $1.82 \%(2.27 \%)$ & 12.00 & 0.0001 \\
ASCH & $41.05 \%(42.20 \%)$ & $42.70 \%(43.29 \%)$ & $1.65 \%(1.09 \%)$ & 7.45 & 0.0001 \\
ASONLY & $27.11 \%(27.42 \%)$ & $24.09 \%(24.02 \%)$ & $-3.02 \%(-3.4 \%)$ & -13.01 & 0.0001 \\
\hline
\end{tabular}

\section{Is the new tick size still the binding constraint on the absolute spread?}

\subsection{Effects of stock characteristics on quote revision behavior}

Although a quote revision can involve changes in the spread, depth, or both, a significant portion of the observed quote revisions involves changes only in either the spread or the depth. [Note that quote revisions that involve changes only in the depth (spread) account for $44.57 \%(8.81 \%)$ of all the quote revisions during the post tick-size change period.] Moreover, there is wide variation across stocks in the proportion of this type of quote revisions. For some stocks, nearly $50 \%$ of the 
observed quote revisions involve changes only in the spread, while other stocks do not exhibit such quote revisions. In this section we examine whether there exists any cross-sectional association between quote revision behavior and stock characteristics. In particular, we analyze whether the proportion of quote revisions involving changes in the spread or depth is related to the attributes of the underlying stocks.

Earlier we noted that the proportion of quote revisions that involve changes in the spread is smaller than the proportion of quote revisions that involve changes in the depth before the tick-size reduction and that the former has gone up significantly since the tick-size reduction. While these results suggest that the minimum price variation was the binding constraint on spreads prior to the tick-size reduction, it is unclear whether the reduced tick size is still the binding constraint. The present analysis helps determine whether the minimum price or size variation imposes any restriction on quote revisions after the tick-size reduction.

While it is difficult to infer whether such a restriction exists from a sequence of quote revisions, we can infer the presence of such a restriction by examining crosssectional correlation between quote revision frequency and stock attributes. The frequency of quote revisions that involve changes in the spread is likely to be greater when the equilibrium spread (i.e., the spread the dealer would have quoted if there were no binding constraint, as determined by the dealer's inventory, adverse selection, and order processing costs) is greater than the minimum price variation. ${ }^{6}$ To the extent that the dealer's market-making costs are correlated with the attributes of the underlying stocks and the equilibrium spread is a function of these attributes, the frequency of quote revisions that involve changes in the spread is likely to be related to these stock attributes. For example, a significant positive correlation between share price and the number of quote revisions that involve changes in the spread may be interpreted as evidence that the minimum price variation is the binding constraint on the spreads of low-price stocks. Similarly, a significant negative correlation between trading volume and the frequency of quote revisions that involve changes in the spread may be interpreted as evidence that the minimum price variation is the binding constraint on the spreads of large-volume stocks (i.e., low-spread stocks).

To examine the relation between quote revision behavior and stock characteristics, we employ four regression models. In the first two models, we regress SPCH and SPONLY, respectively, against four stock attributes - share price (AVGPRC), the number of trades (NTRADE), trade size (AVGTRD), and the standard deviation of daily stock returns (VOLA). In the next two regressions, we use the proportions of quote revisions involving changes in the depth (DPCH and DPONLY) as the dependent variables. We report the regression results from our 2,223 cross-sectional observations in Table 3. Panel A shows the results before the tick-size reduction and panel $\mathrm{B}$ shows the results after the tick-size reduction.

The results show that SPCH and SPONLY are negatively related to NTRADE and AVGTRD, and positively to AVGPRC and VOLA during both periods. In contrast, DPCH and DPONLY are positively related to NTRADE, and negatively

\footnotetext{
${ }^{6}$ Note that the equilibrium spread is not observable.
} 
Table 3

Spread- and depth-quote revisions and stock characteristics

This table shows the results of the following regression models: SPCH $=\beta_{0}+\Sigma \beta_{i} X_{i}+\varepsilon_{1}$; SPONLY $=$ $\beta_{0}+\Sigma \beta_{i} X_{i}+\varepsilon_{2} ; \mathrm{DPCH}=\alpha_{0}+\Sigma \alpha_{i} X_{i}+\varepsilon_{3} ; \mathrm{DPONLY}=\alpha_{0}+\Sigma \alpha_{i} X_{i}+\varepsilon_{4} ;$ and PMIN $=\gamma_{0}+\Sigma \gamma_{i} X_{i}+\varepsilon_{5}$; where SPCH is the proportion of quote revisions involving changes in the spread, SPONLY is the proportion of quote revisions involving changes only in the spread, DPCH is the proportion of quote revisions involving changes in the depth, DPONLY is the proportion of quote revisions involving changes only in the depth, PMIN is the proportion of spread quotes that are equal to the tick size, $X_{i}(i=1-4)$ represents one of the four stock attributes, $\Sigma$ denotes the summation over $i=1-4, \alpha, \beta$, and $\gamma$ are the regression coefficients, and $\varepsilon_{\mathrm{s}}$ are the error terms. AVGPRC is the mean daily closing price, NTRADE is the average daily number of transactions, AVGTRD is the average trade size (in number of shares), and VOLA is the standard deviation of daily returns during each sub-period. We use log of independent variables in the regression. Numbers in parentheses are the absolute values of $t$-statistics.

\begin{tabular}{|c|c|c|c|c|c|c|c|}
\hline & Intercept & AVGPRC & NTRADE & AVGTRD & VOLA & $F$-value & Adj. $R^{2}$ \\
\hline \multicolumn{8}{|c|}{ A. Regression results using data before the tick-size change } \\
\hline $\mathrm{SPCH}$ & $\begin{array}{c}0.9855 \\
(28.29)^{*}\end{array}$ & $\begin{array}{r}0.1459 \\
(38.53)^{*}\end{array}$ & $\begin{array}{l}-0.0973 \\
(37.41)^{*}\end{array}$ & $\begin{array}{c}-0.0385 \\
(9.91)^{*}\end{array}$ & $\begin{array}{r}0.1248 \\
(31.68)^{*}\end{array}$ & $781^{*}$ & 0.584 \\
\hline SPONLY & $\begin{array}{c}0.3454 \\
(21.57)^{*}\end{array}$ & $\begin{array}{c}0.0552 \\
(31.71)^{*}\end{array}$ & $\begin{array}{l}-0.0406 \\
(33.95)^{*}\end{array}$ & $\begin{array}{l}-0.0201 \\
(11.27)^{*}\end{array}$ & $\begin{array}{c}0.0401 \\
(22.16)^{*}\end{array}$ & $620^{*}$ & 0.527 \\
\hline $\mathrm{DPCH}$ & $\begin{array}{r}0.9647 \\
(20.24)^{*}\end{array}$ & $\begin{array}{c}-0.0231 \\
(3.47)^{*}\end{array}$ & $\begin{array}{l}0.0125 \\
(3.51)^{*}\end{array}$ & $\begin{array}{c}-0.0203 \\
(3.81)^{*}\end{array}$ & $\begin{array}{c}-0.0315 \\
(5.85)^{*}\end{array}$ & $22^{*}$ & 0.037 \\
\hline DPONLY & $\begin{array}{l}0.3246 \\
(7.04)^{*}\end{array}$ & $\begin{array}{l}-0.0831 \\
(16.56)^{*}\end{array}$ & $\begin{array}{c}0.0692 \\
(20.09)^{*}\end{array}$ & $\begin{array}{c}-0.0019 \\
(0.36)\end{array}$ & $\begin{array}{l}-0.0532 \\
(10.19)^{*}\end{array}$ & $138^{*}$ & 0.198 \\
\hline PMIN & $\begin{array}{c}-0.0925 \\
(2.26)^{* * *}\end{array}$ & $\begin{array}{l}-0.2643 \\
(59.33)^{*}\end{array}$ & $\begin{array}{c}0.1347 \\
(44.01)^{*}\end{array}$ & $\begin{array}{c}0.0499 \\
(10.93)^{*}\end{array}$ & $\begin{array}{l}-0.1601 \\
(34.52)^{*}\end{array}$ & $1,407^{*}$ & 0.717 \\
\hline \multicolumn{8}{|c|}{ B. Regression results using data after the tick-size change } \\
\hline $\mathrm{SPCH}$ & $\begin{array}{c}1.1303 \\
(35.76)^{*}\end{array}$ & $\begin{array}{c}0.1258 \\
(37.32)^{*}\end{array}$ & $\begin{array}{l}-0.0848 \\
(35.87)^{*}\end{array}$ & $\begin{array}{l}-0.0408 \\
(11.14)^{*}\end{array}$ & $\begin{array}{r}0.1220 \\
(37.29)^{*}\end{array}$ & $920^{*}$ & 0.623 \\
\hline SPONLY & $\begin{array}{r}0.4381 \\
(23.65)^{*}\end{array}$ & $\begin{array}{r}0.0621 \\
(31.42)^{*}\end{array}$ & $\begin{array}{l}-0.0454 \\
(32.81)^{*}\end{array}$ & $\begin{array}{l}-0.0229 \\
(10.68)^{*}\end{array}$ & $\begin{array}{r}0.0500 \\
(26.07)^{*}\end{array}$ & $673^{*}$ & 0.547 \\
\hline $\mathrm{DPCH}$ & $\begin{array}{c}0.7022 \\
(21.55)^{*}\end{array}$ & $\begin{array}{l}-0.0544 \\
(15.65)^{*}\end{array}$ & $\begin{array}{c}0.0438 \\
(17.99)^{*}\end{array}$ & $\begin{array}{c}-0.0056 \\
(1.49)\end{array}$ & $\begin{array}{c}-0.0268 \\
(7.93)^{*}\end{array}$ & $115^{*}$ & 0.170 \\
\hline DPONLY & $\begin{array}{l}0.0100 \\
(0.30)\end{array}$ & $\begin{array}{l}-0.1182 \\
(33.25)^{*}\end{array}$ & $\begin{array}{c}0.0832 \\
(33.39)^{*}\end{array}$ & $\begin{array}{c}0.0123 \\
(3.18)^{*}\end{array}$ & $\begin{array}{l}-0.0987 \\
(28.64)^{*}\end{array}$ & $588^{*}$ & 0.514 \\
\hline PMIN & $\begin{array}{l}-0.6441 \\
(14.14)^{*}\end{array}$ & $\begin{array}{l}-0.2262 \\
(46.54)^{*}\end{array}$ & $\begin{array}{r}0.1285 \\
(37.72)^{*}\end{array}$ & $\begin{array}{r}0.0683 \\
(12.94)^{*}\end{array}$ & $\begin{array}{l}-0.1716 \\
(36.40)^{*}\end{array}$ & $1,180^{*}$ & 0.680 \\
\hline
\end{tabular}

*Significant at the $1 \%$ level.

${ }^{* * *}$ Significant at the $5 \%$ level.

to AVGPRC and VOLA during both periods. The effect of trade size (AVGTRD) on the frequency of depth-quote revisions is weaker than that of other stock attributes. We find that the effect of AVGTRD on DPONLY during the pre tick-size change period and the corresponding effect on DPCH during the post tick-size change period are not statistically significant. These results suggest that specialists 
are more likely to revise spread quotes (with or without changes in depth quotes) for less-traded, high-priced, or riskier stocks. Conversely, they are more likely to revise depth quotes (with or without changes in spread quotes) for stocks with large volume, low price, or low risk.

These results are consistent with the view that the minimum price variation is more likely to be the binding constraint on absolute spreads for high-volume and/or lowprice stocks than for low-volume and/or high-price stocks (see Harris, 1994). For high-volume and/or low-price stocks, there is a high probability that the desired spread is less than the tick size. Specialists are unlikely to change the spread of these stocks because the quoted spread is already greater than the desired spread (thus they do not want to increase the spread) and also because the quoted spread cannot be reduced due to the minimum tick size. Similarly, the minimum price variation is more likely to be the binding constraint for low-risk stocks than high-risk stocks because the spread is positively related to return volatility.

Indeed when we regress the proportion of spread quotes that are equal to the minimum tick size (PMIN) against AVGPRC, NTRADE, AVGTRD, and VOLA, we find (see Table 3 ) that PMIN is positively related to NTRADE and AVGTRD, but negatively to AVGPRC and VOLA. These results are consistent with our conjecture that the minimum price variation is more likely to be the binding constraint on the spread of stocks with large volume, low price, and low return volatility.

The above results suggest that the relative frequency of dealer quote revisions involving changes in the spread or depth is determined by the equilibrium spread and, by implication, its determinants. Although the observed spread is larger than the equilibrium spread whenever the minimum price variation is the binding constraint on the latter, these two spread measures are likely to be highly correlated across stocks. Consequently, the relative frequency of quote revisions is also likely to be related to the observed spread. Indeed, when we re-estimate the above regression models after we replace the four stock attributes with the average observed spread, we find that the frequency of spread (depth) usage is significantly and positively (negatively) related to the average observed spread. ${ }^{7}$

Although the specialists' use of price quotes vis-a-vis quantity quotes has risen after the tick-size reduction, they still revise depth quotes more frequently than spread quotes. Because the regression results from data after the tick-size change are qualitatively identical to those from data before the tick-size reduction, the minimum price variation appears to influence the relative frequency of the spread and depth, even with the smaller tick size. Overall, these results suggest that the minimum price variation (i.e., \$1/16) still poses a restriction on specialist quotes.

\subsection{Effects of the tick size and stock characteristics on quote revision behavior}

Earlier we presented evidence that the proportion of quote revisions involving changes only in the depth (DPONLY) declined significantly after the tick-size

\footnotetext{
${ }^{7}$ The results are available from the authors upon request.
} 
reduction. We also find a significant increase in the proportion of quote revisions involving changes in the spread (SPCH and SPONLY). Given our results that SPCH, SPONLY, and DPONLY are strongly related to stock attributes, however, it is possible that the observed changes in these variables may be due to changes in the stock attributes between the two periods, rather than due to the change in tick size per se. Similarly, the observed decline in PMIN after the tick-size reduction may also be due to changes in these stock attributes. To examine whether the observed changes in SPCH, SPONLY, DPONLY, and PMIN are due to the changes in the stock attributes, we estimate the following regression models:

$$
\begin{aligned}
& \mathrm{SPCH}^{\text {post }}-\mathrm{SPCH}^{\text {pre }}=\beta_{0}+\Sigma \beta_{i}\left(X_{i}^{\text {post }}-X_{i}^{\text {pre }}\right)+\varepsilon_{1} ; \\
& \text { SPONLY } \\
& \text { DPost }-S P O N L Y \text { pre }=\beta_{0}+\Sigma \beta_{i}\left(X_{i}^{\text {post }}-X_{i}^{\text {pre }}\right)+\varepsilon_{2} ; \\
& \text { PMIN }^{\text {post }}-\text { PMIN }^{\text {pre }}=\gamma_{0}+\sum \gamma_{i}\left(X_{i}^{\text {post }}-X_{i}^{\text {pre }}\right)+\varepsilon_{4} ;
\end{aligned}
$$

where superscripts 'post' and 'pre' denote, respectively, the post and pre tick-size change values of the variables, $X_{i}(i=1-4)$ represents one of the four stock attributes, $\Sigma$ denotes the summation over $i=1-4, \alpha, \beta$, and $\gamma$ are the regression coefficients, and $\varepsilon_{\mathrm{S}}$ are the error terms. The results of these regressions help determine whether there exists any difference in SPCH, SPONLY, DPONLY, and PMIN between the pre and post tick-size change periods, after controlling for changes in stock attributes between the two periods.

We report the regression results in Table 4 (panel A). The highly significant and positive intercept $\left(\beta_{0}\right)$ in regression models (1) and (2) suggests that the proportion of quote revisions that involve changes in the spread (SPCH and SPONLY) increased after the tick-size reduction and this increase is not due to changes in stock attributes. We note that the estimated intercepts $(0.1211$ and 0.0333$)$ are only slightly smaller than the corresponding figure $(12.54 \%$ and $3.49 \%)$ in Table 2, indicating that the observed increase in SPCH and SPONLY is largely due to factors other than changes in stock attributes.

The highly significant and negative intercept $\left(\alpha_{0}\right)$ in regression model (3) suggests that the proportion of quote revisions involving changes only in the depth (DPONLY) declined after the tick-size change and this decline cannot be attributed to differences in stocks attributes between the two periods. Note that our estimate $(-0.0778)$ of $\alpha_{0}$ is only slightly smaller (in absolute value) than the corresponding figure $(-7.91 \%)$ in Table 2 . This suggests that most of the observed decline in DPONLY is due to factors other than changes in stock attributes.

Finally the highly significant and negative intercept $\left(\gamma_{0}\right)$ in regression model (4) suggests that the proportion of quotes that are equal to the tick size (PMIN) declined after the tick-size change and this decline cannot be attributed to changes in stock attributes. We note that the estimated intercept $(-0.2668)$ is only slightly smaller (in absolute value) than the corresponding figure $(-27.36 \%)$ in Table 2 , indicating that 
Table 4

Regression results using differences in the variables between before and after the tick-size change

Panel A shows the results of the following models: $\mathrm{SPCH}{ }^{\text {post }}-\mathrm{SPCH}^{\text {pre }}=\beta_{0}+\Sigma \beta_{i}\left(X_{i}^{\text {post }}-X_{i}^{\text {pre }}\right)+\varepsilon_{1}$; SPONLY $^{\text {post }}-$ SPONLY $^{\text {pre }}=\beta_{0}+\Sigma \beta_{i}\left(X_{i}^{\text {post }}-X_{i}^{\text {pre }}\right)+\varepsilon_{2} ; \quad$ DPONLY $^{\text {post }}-$ DPONLY $^{\text {pre }}=\alpha_{0}+\sum \alpha_{i}$ $\left(X_{i}^{\text {post }}-X_{i}^{\text {pre }}\right)+\varepsilon_{3}$; and PMIN ${ }^{\text {post }}-$ PMIN $^{\text {pre }}=\gamma_{0}+\sum \gamma_{i}\left(X_{i}^{\text {post }}-X_{i}^{\text {pre }}\right)+\varepsilon_{4}$. Panel B shows the results of the following models: $\mathrm{SPCH}{ }^{\text {post }}-\mathrm{SPCH}^{\text {pre }}=\beta_{0}+\Sigma \beta_{i}\left(X_{i}^{\text {post }}-X_{i}^{\text {pre }}\right)+\beta_{5}\left(\mathrm{DEPTH}^{\text {post }}-\mathrm{DEPTH}^{\text {pre }}\right)$ $+\varepsilon_{1}$; and SPONLY $^{\text {post }}-$ SPONLY $^{\text {pre }}=\beta_{0}+\Sigma \beta_{i}\left(X_{i}^{\text {post }}-X_{i}^{\text {pre }}\right)+\beta_{5}\left(\right.$ DEPTH $^{\text {post }}-$ DEPTH $\left.^{\text {pre }}\right)+\varepsilon_{2}$. Superscripts 'post' and 'pre' denote, respectively, the post and pre tick-size change values of the variables, SPCH is the proportion of quote revisions involving changes in the spread, SPONLY is the proportion of quote revisions involving changes only in the spread, DPONLY is the proportion of quote revisions involving changes only in the depth, PMIN is the proportion of spread quotes that are equal to the tick size, $X_{i}(i=1-4)$ represents one of the four stock attributes, $\Sigma$ denotes the summation over $i=1-4, \alpha, \beta$, and $\gamma$ are the regression coefficients, and $\varepsilon_{s}$ are the error terms. AVGPRC is the mean daily closing price, NTRADE is the average daily number of transactions, AVGTRD is the average trade size (in number of shares), VOLA is the standard deviation of daily returns, and DEPTH is the average quoted depth during each sub-period. We use log of independent variables in the regression. Numbers in parentheses are the absolute values of $t$-statistics.

Intercept $\triangle$ AVGPRC $\triangle$ NTRADE $\triangle$ AVGTRD $\triangle$ VOLA $\triangle$ DEPTH $F$-value Adj. $R^{2}$

A. Changes in quote revisions as a function of changes in stock attributes

\begin{tabular}{|c|c|c|c|c|c|c|c|}
\hline$\triangle \mathrm{SPCH}$ & $\begin{array}{r}0.1211 \\
(72.34)^{*}\end{array}$ & $\begin{array}{c}0.0305 \\
(2.61)^{*}\end{array}$ & $\begin{array}{c}-0.0121 \\
(2.47)^{*}\end{array}$ & $\begin{array}{l}-0.0452 \\
(11.24)^{*}\end{array}$ & $\begin{array}{c}0.0223 \\
(8.05)^{*}\end{array}$ & $55.17^{*}$ & 0.089 \\
\hline$\triangle$ SPONLY & $\begin{array}{r}0.0333 \\
(33.65)^{*}\end{array}$ & $\begin{array}{c}0.0432 \\
(6.26)^{*}\end{array}$ & $\begin{array}{c}-0.0174 \\
(6.00)^{*}\end{array}$ & $\begin{array}{c}-0.0218 \\
(9.18)^{*}\end{array}$ & $\begin{array}{c}0.0146 \\
(8.96)^{*}\end{array}$ & $62.88^{*}$ & 0.100 \\
\hline$\triangle \mathrm{DPONLY}$ & $\begin{array}{l}-0.0778 \\
(28.38)^{*}\end{array}$ & $\begin{array}{c}-0.0604 \\
(3.15)^{*}\end{array}$ & $\begin{array}{c}0.0405 \\
(5.04)^{*}\end{array}$ & $\begin{array}{c}0.0295 \\
(4.49)^{*}\end{array}$ & $\begin{array}{l}-0.0476 \\
(10.50)^{*}\end{array}$ & $38.39^{*}$ & 0.063 \\
\hline$\Delta \mathrm{PMIN}$ & $\begin{array}{l}-0.2668 \\
(94.42)^{*}\end{array}$ & $\begin{array}{c}-0.1244 \\
(6.31)^{*}\end{array}$ & $\begin{array}{c}0.0400 \\
(4.83)^{*}\end{array}$ & $\begin{array}{r}0.0703 \\
(10.36)^{*}\end{array}$ & $\begin{array}{c}-0.0354 \\
(7.57)^{*}\end{array}$ & $62.72^{*}$ & 0.100 \\
\hline
\end{tabular}

B. Changes in spread-quote revisions as a function of changes in stock attributes and depths

\begin{tabular}{lcccccccc}
$\Delta \mathrm{SPCH}$ & 0.0898 & 0.0137 & 0.0052 & 0.0131 & 0.0192 & -0.886 & $265.17^{*}$ & 0.373 \\
& $(52.74)^{*}$ & $(1.41)$ & $(1.26)$ & $(3.44)^{*}$ & $(8.38)^{*}$ & $(31.71)^{*}$ & & \\
\multirow{2}{*}{$\Delta \mathrm{SPONLY}$} & 0.0277 & 0.0402 & -0.0143 & -0.0113 & 0.0141 & -0.0159 & $64.88^{*}$ & 0.126 \\
& $(23.14)^{*}$ & $(5.90)^{*}$ & $(4.96)^{*}$ & $(4.25)^{*}$ & $(8.74)^{*}$ & $(8.10)^{*}$ & &
\end{tabular}

* Significant at the $1 \%$ level.

the observed decline in PMIN is largely due to factors other than changes in stock attributes.

Overall, these results indicate that the observed changes in SPCH, SPONLY, DPONLY, and PMIN are largely due to factors other than changes in stock attributes. It is our conjecture that one such factor is that smaller tick size reduces the likelihood of minimum price variation being the binding constraint on the specialists' spread quotes. Because the smaller tick size poses less restriction on their spread quote decisions, NYSE specialists utilize spread (depth) quotes more (less) frequently for their liquidity management. 
Although our results are generally consistent with our conjecture that the relative usage of spread and depth quotes is largely determined by whether the minimum price variation is the binding constraint, there are other possible explanations for the observed empirical regularity. For example, the negative relation between the frequency of spread revisions and the number of trades in both periods may simply reflect the fact that actively traded stocks tend to have larger depths (relative to the average trade size) and bigger books. Consequently, a larger fraction of trades will pick off shares at a particular price without an accompanying change in the spread.

Similarly, the fact that the frequency of spread-quote revisions increases following the reduction in tick size is consistent with the lower quoted and limit order book depth found by Goldstein and Kavajecz (2000), since fewer trades are needed to exhaust the available depth at each price. Indeed, if a decrease in depth were driving the increased frequency of spread revisions, then we would expect the increase to be more pronounced when the decrease in depth is larger. To examine this possibility, we estimate the following regression models:

$$
\begin{aligned}
\mathrm{SPCH}^{\text {post }}-\mathrm{SPCH}^{\mathrm{pre}}= & \beta_{0}+\Sigma \beta_{i}\left(X_{i}^{\text {post }}-X_{i}^{\text {pre }}\right) \\
& +\beta_{5}\left(\mathrm{DEPTH}^{\text {post }}-\mathrm{DEPTH}^{\text {pre }}\right)+\varepsilon_{1} ;
\end{aligned}
$$

and

$$
\begin{aligned}
\text { SPONLY }^{\text {post }}-\text { SPONLY }^{\text {pre }}= & \beta_{0}+\sum \beta_{i}\left(X_{i}^{\text {post }}-X_{i}^{\text {pre }}\right) \\
& +\beta_{5}\left(\text { DEPTH }^{\text {post }}-\text { DEPTH }^{\text {pre }}\right)+\varepsilon_{2} ;
\end{aligned}
$$

where DEPTH is the average quoted depth during each period and all other variables are the same as previously defined.

The regression results (see panel B, Table 4) show that changes in both SPCH and SPONLY are significantly and negatively related to changes in DEPTH, indicating that increases in the frequency of spread revisions are greater for those stocks that exhibited greater decreases in quoted depths after the tick-size reduction. Hence, the greater frequency of spread-quote revisions after the tick-size change can be attributed, at least in part, to the smaller quoted depth. Note, however, that the estimated intercepts (0.0898 and 0.0277) in both equations are highly significant and positive even after we control for the effect of depth changes on the frequency of spread-quote revisions. Hence, the observed increase in the frequency of spread quote cannot be attributed entirely to the smaller quoted depth after the tick-size reduction.

\section{Intraday variations in quote revision}

In this section we examine the intraday pattern of the specialist's quote revisions. While previous studies have also analyzed intraday data for NYSE stocks, the main focus of those studies has been the analysis of intraday variations in spreads, depths, volatility, and volume (see, e.g., Foster and Viswanathan, 1993; Lee et al., 1993; 


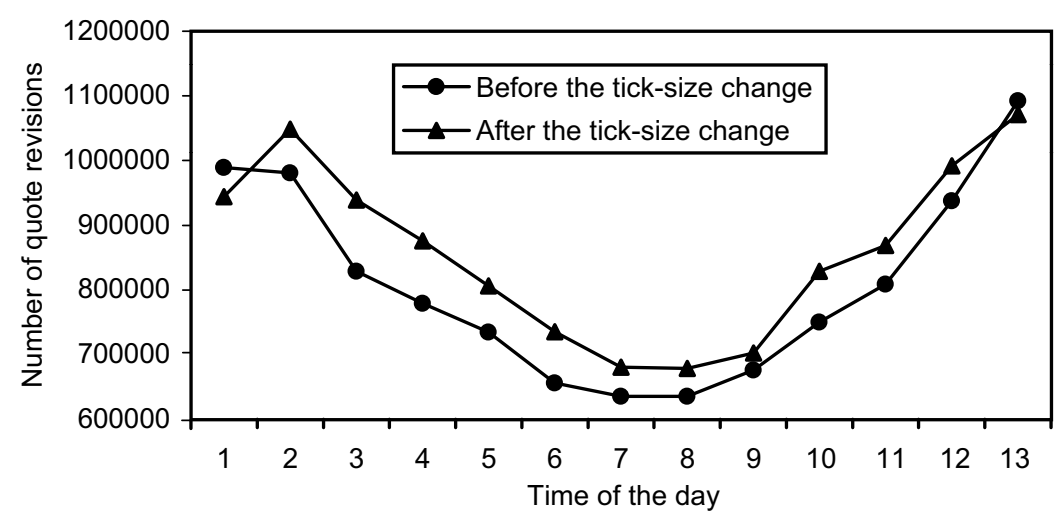

Fig. 1. Intraday variation in the number of quote revisions.

Chan et al., 1995). In contrast, our focus here is to examine intraday variation in the number of quote revisions. To the extent that quote revisions are motivated by changes in information or trading desire, the present analysis can shed additional light on the adverse selection and inventory models of market making.

We partition each day into 13 successive 30-min intervals and calculate the number of quote revisions during each interval. Fig. 1 shows the number of quote revisions during each interval for our entire sample of 2,223 stocks. Intraday variation in the number of quote revisions follows the U-shaped pattern during both the pre and post tick-size change periods. To the extent that quote revisions are prompted by transactions, the frequency of quote revisions is likely to be correlated with trading volume. Hence, the U-shaped pattern of the intraday quote revision frequency is likely to be driven in part by the corresponding pattern in trading volume, which has been shown in previous studies (see, e.g., Foster and Viswanathan, 1993; Chan et al., 1995). ${ }^{8}$ Because the rate of information resolution is greater during the early hours of trading than midday, we expect the number of information-driven quote revisions to be greater during this period. Hence, frequent quote revisions during early hours of trading may also reflect the specialists' and other liquidity providers' attempt to deal with adverse selection problems. The large number of quote revisions during the last hour of trading is consistent with inventory models of market making. Amihud and Mendelson (1982) suggest that risk-averse dealers want to end the trading day with the desired level of inventory and thus they may actively seek order flow before the close in an attempt to resolve inventory imbalances accumulated during the day. The large number of quote revisions during the last hour of trading may reflect the market makers' attempt to seek desired order flows.

\footnotetext{
${ }^{8}$ Chung et al. (1999) show that the number of limit order placements follows the U-shaped intraday pattern. Because specialists are obligated to display quote-matching and quote-improving orders, intraday variation in the number of quote revisions is likely to mirror intraday variation in the number of limit order submissions. Consequently, the intraday pattern of quote revision frequencies may also reflect intraday variation in limit orders.
} 


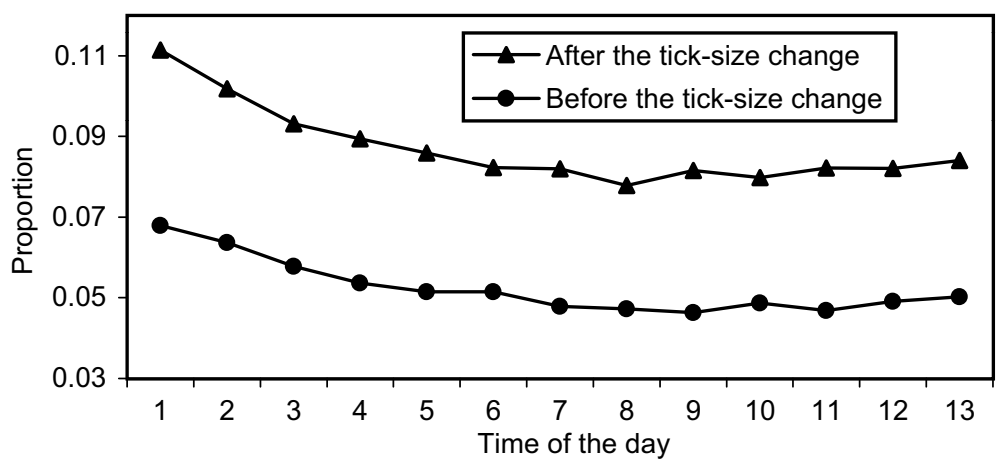

Fig. 2. Intraday variation in the proportion of quote revisions involving changes only in spreads (i.e., QRCs $(-, 0)$ and $(+, 0))$.

Fig. 2 shows intraday variation in the proportion of quote revisions involving changes only in the spread [i.e., QRCs $(-, 0)$ and $(+, 0)$ ] and Fig. 3 shows intraday variation in the proportion of quote revisions involving changes only in the depths [i.e., QRCs $(0,-)$ and $(0,+)$ ]. These figures show that the proportion of quote revisions involving changes in spread is highest during the early hour of trading. In contrast, the proportion of quote revisions that involve changes in the depth is lowest during the early hour of trading. One possible explanation for these results is that the minimum price variation is less likely to be the binding constraint during the early hour of trading as the spread is wider during this period (see Brock and Kleidon, 1992; McInish and Wood, 1992; Lee et al., 1993; Chung et al., 1999). ${ }^{9}$ As a result, liquidity providers revise their price quotes more freely during early hours of trading than other times of the day.

More importantly, we note that intraday variation in the proportion of quote revisions involving changes in spread (depth) during the post tick-size change period is quite similar to intraday variation during the pre tick-size change period, although there are parallel shifts in both curves. This result provides additional support for our earlier observation that the minimum price variation might still be a limiting constraint on specialist quotes even after the tick-size reduction. ${ }^{10}$

\footnotetext{
${ }^{9}$ When we replicate these studies using our data, we find intraday variations in the spread and depth that are similar to those reported in these studies during both the pre and post tick-size reduction periods.

${ }^{10}$ To the extent that NYSE specialists revise their quotes in response to transactions, the U-shaped variation in the number of quote revisions is likely to be driven in part by the corresponding variation in trading volume. We find that intraday variation in quote revisions that do not involve transactions is similar to the pattern reported in Fig. 1. Hence the observed U-shaped intraday variation in the number of quote revisions is not entirely due to the intraday trading pattern. As in Figs. 2 and 3, we also find that the proportion of quote revisions involving changes in the spread (depth) is highest (lowest) during the early hour of trading during both the pre and post tick-size change periods. This result corroborates our earlier finding that the minimum price variation is still a binding constraint on quote revisions even after the ticksize reduction.
} 


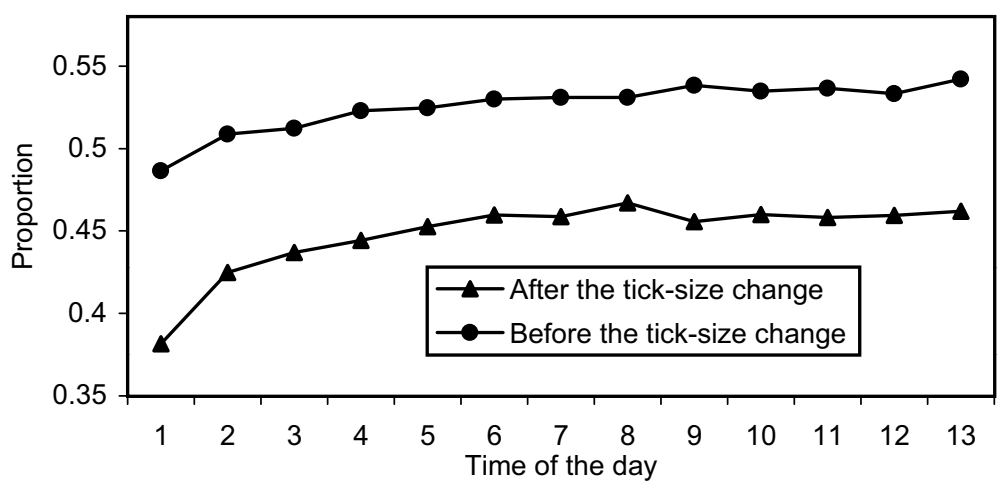

Fig. 3. Intraday variation in the proportion of quote revisions involving changes only in depths (i.e., QRCs $(0,-)$ and $(0,+))$.

\section{Evidence after decimalization}

Our results from both cross-sectional and intraday data reported in the previous sections suggest that the minimum price variation of $\$ 1 / 16$ was the binding constraint on specialists' spread- and price-quote decisions. The results suggest that NYSE specialists and other liquidity providers are likely to change spread and price quotes more frequently for liquidity management once the binding constraint is removed. The NYSE started to implement decimal pricing in August 2000. The conversion to decimal trading began with seven listed issues on August 28 and subsequently extended to 57 additional stocks on September 25 and to 94 more issues on December 4. All NYSE-listed issues began trading in decimals on January 29, 2001. The phased-in approach allowed all trading posts on the NYSE floor to gain experience in trading decimal-priced stocks.

In this section, we analyze the quote revision behavior of NYSE specialists and other liquidity providers after decimalization. Specifically, we use data for the first three batches of NYSE issues (i.e., 158 issues) that were subject to decimal pricing by December 4, 2000.

To examine whether decimal pricing has changed the quote revision behavior on the NYSE, we calculate the frequency of spread- and depth-quote revisions for each QRC using data before and after decimalization for these stocks. We use January 2001 TAQ data to calculate the frequency of quote revisions for each QRC after decimalization and match the result with the corresponding figure before decimalization for each stock in our study sample. We then aggregate the frequency across stocks to obtain the mean and median values of SPCH, SPONLY, DPCH, and DPONLY. To examine whether decimalization affected price rigidity, we also calculate the mean and median values of the proportions of price- and size-quote revisions (BPCH, BPONLY, BSCH, BSONLY, APCH, APONLY, ASCH, and ASONLY) using the same method.

We show the results in Table 5. Note that the relative frequencies of quote revisions that involve changes in the spread $(\mathrm{SPCH}=48.03 \%$ and 
Table 5

Comparison of quote revision behavior before and after decimalization

This table shows the mean (median) values of SPCH, SPONLY, DPCH, DPONLY, BPCH, BPONLY, BSCH, BSONLY, APCH, APONLY, ASCH, and ASONLY before and after decimalization and the results of paired comparison $t$-tests on the equality of the mean between the two periods. SPCH is the proportion of quote revisions involving changes in the spread, $\mathrm{DPCH}$ is the proportion of quote revisions involving changes in the depth, SPONLY is the proportion of quote revisions involving changes only in the spread, and DPONLY is the proportion of quote revisions involving changes only in the depth. Similarly, $\mathrm{BPCH}(\mathrm{APCH})$ is the proportion of quote revisions involving changes in the bid (ask) price, $\mathrm{BSCH}$ $(\mathrm{ASCH})$ is the proportion of quote revisions involving changes in the bid (ask) size, BPONLY (APONLY) is the proportion of quote revisions involving changes only in the bid (ask) price, and BSONLY (ASONLY) is the proportion of quote revisions involving changes only in the bid (ask) size.

\begin{tabular}{|c|c|c|c|c|c|}
\hline & Before decimalization & After decimalization & $\begin{array}{l}\text { Difference } \\
\text { (after - before) }\end{array}$ & $t$-value & $p$-value \\
\hline \multicolumn{6}{|c|}{ A. Spread-depth revisions } \\
\hline SPCH & $40.82 \%(41.34 \%)$ & $48.03 \%(46.28 \%)$ & $7.21 \%(4.94 \%)$ & 4.02 & 0.0001 \\
\hline SPONLY & $9.34 \%(8.32 \%)$ & $14.06 \%(11.46 \%)$ & $4.72 \%(3.14 \%)$ & 3.50 & 0.0006 \\
\hline $\mathrm{DPCH}$ & $76.04 \%(77.10 \%)$ & $67.41 \%(68.97 \%)$ & $-8.63 \%(-8.13 \%)$ & -5.51 & 0.0001 \\
\hline DPONLY & $44.55 \%(43.14 \%)$ & $33.44 \%(33.18 \%)$ & $-11.11 \%(-9.96 \%)$ & -6.35 & 0.0001 \\
\hline \multicolumn{6}{|c|}{ B. Bid price-bid depth revisions } \\
\hline $\mathrm{BPCH}$ & $24.39 \%(24.74 \%)$ & $28.73 \%(26.71 \%)$ & $4.34 \%(1.97 \%)$ & 3.47 & 0.0007 \\
\hline BPONLY & $6.62 \%(5.81 \%)$ & $9.25 \%(6.65 \%)$ & $2.63 \%(0.84 \%)$ & 2.76 & 0.0065 \\
\hline $\mathrm{BSCH}$ & $39.95 \%(39.85 \%)$ & $37.04 \%(37.93 \%)$ & $-2.91 \%(-1.92 \%)$ & -2.97 & 0.0035 \\
\hline BSONLY & $22.17 \%(21.30 \%)$ & $17.56 \%(17.99 \%)$ & $-4.61 \%(-3.31 \%)$ & -4.52 & 0.0001 \\
\hline \multicolumn{6}{|c|}{ C. Ask price-ask depth revisions } \\
\hline $\mathrm{APCH}$ & $25.18 \%(24.43 \%)$ & $27.99 \%(26.20 \%)$ & $2.81 \%(1.77 \%)$ & 2.09 & 0.0390 \\
\hline APONLY & $6.61 \%(5.05 \%)$ & $9.07 \%(6.25 \%)$ & $2.46 \%(1.20 \%)$ & 2.47 & 0.0144 \\
\hline $\mathrm{ASCH}$ & $42.76 \%(43.58 \%)$ & $36.93 \%(37.16 \%)$ & $-5.83 \%(-6.42 \%)$ & -5.20 & 0.0001 \\
\hline ASONLY & $24.19 \%(23.58 \%)$ & $18.00 \%(18.16 \%)$ & $-6.19 \%(-5.42 \%)$ & -5.61 & 0.0001 \\
\hline
\end{tabular}

SPONLY $=14.06 \%$ ) after decimalization are significantly greater than the corresponding figures $(40.82 \%$ and $9.34 \%$ ) before decimalization. Decimal pricing resulted in an increase of $7.21 \%$ in the proportion of quote revisions that involve changes in the spread and an increase of $4.72 \%$ in the proportion of quote revisions that involve changes in the spread alone. In contrast, decimalization led to a significant decrease in the proportion of quote revisions involving changes in the depth. Notice that the proportion of quote revisions that involve changes only in the depth (DPONLY) decreased from $44.55 \%$ to $33.44 \%$ after decimalization. We find similar results from the price- and size-quote revisions. On both the ask and bid sides, we find the proportion of quote revisions involving price changes increased significantly and the proportion of quote revisions involving depth changes decreased significantly.

Overall, these results are consistent with our expectation that the smaller price grid gives liquidity providers more flexibility in their price quotes and thus decimal 
pricing is likely to result in smaller price rigidity and greater price competition. Indeed, early indications show that investors are benefiting from decimal pricing. Chakravarty and Wood (2000) analyze the trading data for the seven securities in the NYSE's August 28 pilot for the first 10 days of trading and find that the bid-ask spreads shrank $38 \%$ on average. The authors also show that for the two-week period following the September 25 pilot the spreads for five of the NYSE's most actively traded stocks dropped $31 \%$ after their conversion to decimals.

\section{Concluding remarks}

Any regulatory or institutional constraints that unduly limit and impede liquidity providers' quote decisions are likely to obstruct the efficiency and competitiveness of the market. In particular, limiting the market maker's ability to use fine price grids may deleteriously affect the liquidity of large-volume and/or low-price stocks. Although the tick-size reduction on June 24, 1997 gave NYSE specialists and other liquidity providers more flexibility in their quote decisions, it is unclear whether the reduced tick size $(\$ 1 / 16)$ was small enough for them to quote their desired prices. According to our findings, specialists and other liquidity providers on the NYSE rely much less on price quotes than size quotes for low-price, high-volume, and low-risk stocks, even after the tick size is reduced to $\$ 1 / 16$. Similarly, we find that the number of quote revisions that involve changes in the spread (depth) is largest (smallest) during the early hour of trading even after the tick-size reduction. These results suggest that the minimum price variation of $\$ 1 / 16$ was still a constraining factor in the price discovery process on the NYSE.

The NYSE began implementing decimal pricing for select stocks from August 28, 2000. Some suggest that a smaller price increment would shift power from public traders to professional traders by making it easier for professionals to step in front of public limit orders. As a result, public traders will display their orders less frequently and switch from limit order strategies to market order strategies. Others argue that decimal prices are easier to use than fractional prices and that a smaller price increment encourages price competition and narrow bid-ask spreads. The results of the present study suggest that decimalization is likely to reduce price rigidity and increases price competition. Consistent with this expectation, we find that the relative frequency of spread- and price-quote revisions increased significantly after the implementation of decimal pricing on the NYSE.

Although this study finds evidence that the relative use of the spread and depth by liquidity providers is strongly correlated to select stock attributes, there are many unanswered questions that remain to be explored. For example, while the results of this study show that specialists tend to change their depth quotes more frequently than spread quotes, we have not established the intertemporal link between these quote changes and concurrent shocks such as changes in trade size, price volatility, and the number of trades. Establishing such a link would be an interesting area for future study. Another fruitful area for future research may be the comparative analysis of quote revision behavior between NYSE specialists and Nasdaq dealers. 
Empirical evidence regarding these issues will no doubt shed further light on the behavior of market makers.

\section{References}

Ahn, H., Cao, C., Choe, H., 1996. Tick size, spread, and volume. Journal of Financial Intermediation 5, $2-22$.

Amihud, Y., Mendelson, H., 1982. Asset pricing behavior in a dealership market. Financial Analysts Journal 38, 50-59.

Bacidore, J., 1997. The impact of decimalization on market quality: an empirical investigation of the Toronto Stock Exchange. Journal of Financial Intermediation 6, 92-120.

Bollen, N., Whaley, R., 1998. Are "teenies" better? Journal of Portfolio Management 25, 10-24.

Brock, W., Kleidon, A., 1992. Periodic market closure and trading volume: a model of intraday bids and asks. Journal of Economic Dynamics and Control 16, 451-489.

Chakravarty, S., Wood, R., 2000. The effect of decimal trading on market liquidity. Working paper, University of Memphis.

Chan, K., Chung, P., Johnson, H., 1995. The intraday behavior of bid-ask spreads for NYSE stocks and CBOE options. Journal of Financial and Quantitative Analysis 30, 329-346.

Chung, K., Van Ness, B., Van Ness, R., 1999. Limit orders and the bid-ask spread. Journal of Financial Economics 53, 255-287.

Foster, F., Viswanathan, S., 1993. Variations in trading volume, return volatility, and trading costs: Evidence on recent price formation models. Journal of Finance 48, 187-211.

Goldstein, M., Kavajecz, K., 2000. Eighths, sixteenths and market depth: changes in tick size and liquidity provision on the NYSE. Journal of Financial Economics 56, 125-149.

Griffiths, M., Smith, B., Turnbull, D., White, R., 1998. The role of tick size in upstairs trading and downstairs trading. Journal of Financial Intermediation 7, 393-417.

Harris, L., 1994. Minimum price variations, discrete bid-ask spreads, and quotation sizes. Review of Financial Studies 7, 149-178.

Harris, L., Hasbrouck, J., 1996. Market vs. limit orders: the SuperDot evidence on order submission strategy. Journal of Financial and Quantitative Analysis 31, 213-231.

Huang, R., Stoll, H., 1996. Dealer versus auction markets: a paired comparison of execution costs on NASDAQ and the NYSE. Journal of Financial Economics 41, 313-357.

Kavajecz, K., 1996. A specialist's quoted depth as a strategic choice variable. Working Paper, University of Pennsylvania.

Kavajecz, K., 1999. A specialist's quoted depth and the limit order book. Journal of Finance 54, 747-771.

Kavajecz, K., Odders-White, E., 1999. An examination of changes in specialist's posted price schedules. Working Paper, University of Pennsylvania.

Lee, C., Mucklow, B., Ready, M., 1993. Spreads, depths, and the impact of earnings information: an intraday analysis. Review of Financial Studies 6, 345-374.

McInish, T., Wood, R., 1992. An analysis of intraday patterns in bid/ask spreads for NYSE stocks. Journal of Finance 47, 753-764.

Porter, D., Weaver, D., 1997. Decimalization and market quality. Financial Management 26, 5-26.

Simaan, Y., Weaver, D., Whitcomb, D., 1998. The quotation behavior of ECNs and Nasdaq market makers. Working Paper, Baruch College, CUNY.

Van Ness, R., Van Ness, B., Pruitt, S., 2000. The impact of the reduction in tick increments in major U.S. markets on spreads, depth, and volatility. Review of Quantitative Finance and Accounting 15, $153-167$.

Ye, J., 1995. Bid-ask prices and sizes: the specialist's optimal quotation strategy. Working Paper, University of Southern California. 